\title{
Precision 96-Channel Dispenser for Microchemical Techniques
}

\author{
J. Stanchfield, D. Wright, S. Hsu ${ }^{1}$, M. Lamsa ${ }^{2}$ and A. Robbins \\ Robbins Scientific, Sunnyvale, CA, ${ }^{1}$ America Red Cross, Philadelphia, PA and ${ }^{2}$ Novo Nordisk Biotech, \\ Davis, CA, USA
}

BioTechniques 20:292-296(February 1996)

\begin{abstract}
A new automated 96-channel microdispenser is described for precise, high-speed dispensing of microliter volumes of reagents. The Hydra-96 ${ }^{\mathrm{TM}}$ is a programmable instrument composed of 96 glass syringes arrayed in a microplate format that fills and dispenses in unison under computer control. Studies show the instrument has less than a $2 \%$ coefficient of variation $(\mathrm{CV})$ across the syringe array when dispensing between 0.5 and $20.0 \mu \mathrm{L}$ of reagent. Blot hybridization studies demonstrate a simple rinsing protocol using $2 \%$ bleach that efficiently cleans the system of DNA without affecting subsequent PCRs. Current uses of the instrument in assembling microassays used in large-scale genetic mapping and sequencing projects and compound library screening are discussed.
\end{abstract}

\section{INTRODUCTION}

Sample amplification techniques, such as polymerase chain reaction (PCR), coupled with improved signal enhancement and detection methods, have led to a new generation of microassays. In addition, screening of large libraries of compounds to identify drug candidates has led to new requirements for rapid, parallel preparation of various enzymelinked immunosorbent assays (ELISA) and bioassays. In laboratory situations where a multitude of small volume reactions are prepared in parallel, robotic multichannel dispensers are normally used. Many commercial systems have limited precision when dispensing below $10 \mu \mathrm{L}$ and have high, fixed operating expenses from the use of disposable tips. Also, robotic systems tend to be inefficient when used for assembling identical reaction mixtures used in many screening-type applications.

The Hydra-96 $96^{\mathrm{TM}}$ Channel Microdispenser was designed for special applications where multiple, identical small-volume reaction mixtures are prepared in microplate format. It provides high speed and precise delivery of reagents down to
$0.5 \mu \mathrm{L}$ using an array of 96 glass syringes that fill and dispense in unison. All instrument operations such as fill and dispense volumes are controlled by an integral microprocessor that has desired parameters stored in nonvolatile memory.

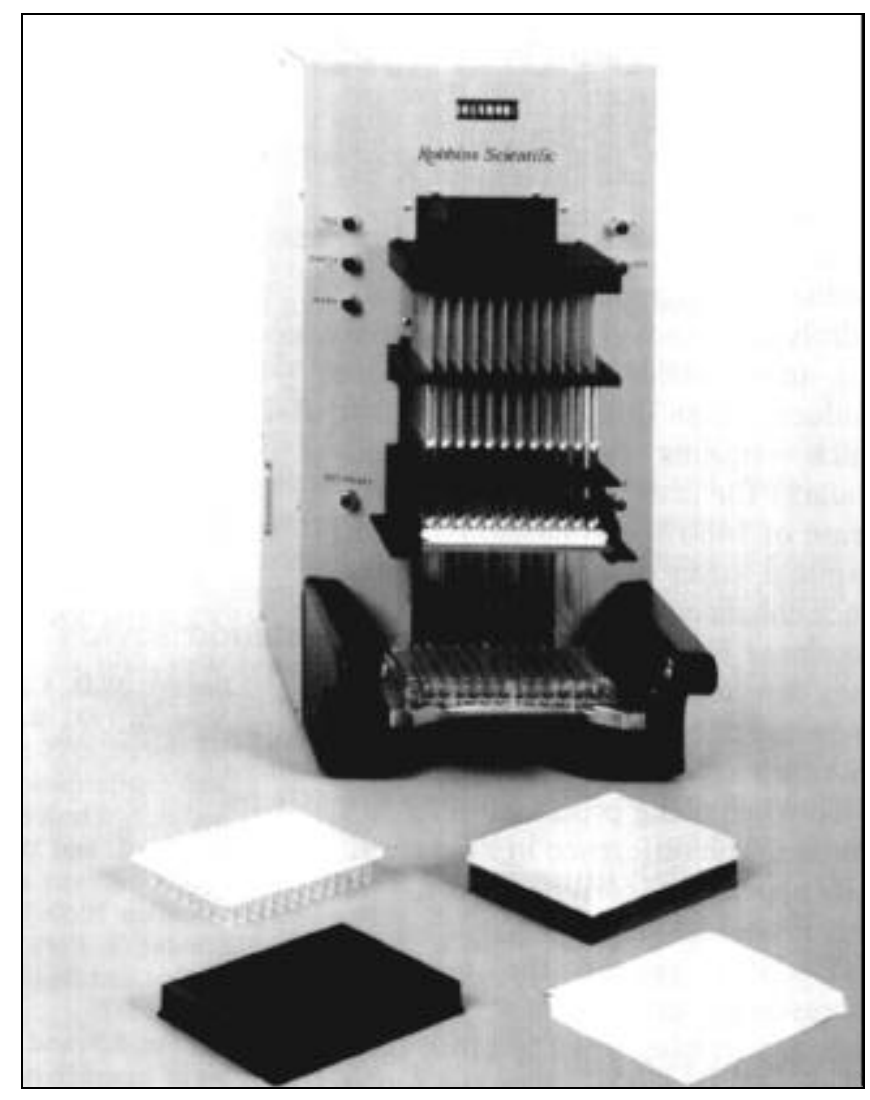

Figure 1. The Hydra-96 Microdispenser. The unit pictured is being used to dispense microliter volumes of reagents from its array of 96 syringes into a microplate. 
The instrument can also be controlled through an external computer when used in conjunction with other automated laboratory equipment.

In this report, results are provided from studies designed to measure the dispensing precision or uniformity of the syringe array. Results from other studies are also presented that demonstrate that simple rinsing of the syringes with bleach and distilled water effectively removes residual DNA without affecting subsequent PCRs. Current applications of the instrument in the human genome project and for combinatorial library screening will be discussed.

\section{MATERIALS AND METHODS}

\section{Instrument Construction and Operation (Figure 1)}

The Hydra-96 Channel Microdispenser (Robbins Scientific, Sunnyvale, CA, USA) is constructed of 96 glass syringes vertically mounted in a bracket that spaces them precisely 9 $\mathrm{mm}$ center to center (two syringe sizes are available: 290 and $580 \mu \mathrm{L}$ ). The up-and-down motion of the syringe plungers for filling and emptying syringes is achieved by the action of a precision screw and stepper motor that moves a plate to which the plungers are attached. The motion of this system is accurate to $2.5 \mu \mathrm{m}$. Syringe needles two inches in length are Teflon ${ }^{\circledR}$-coated on the outside at their tips to ensure uniform drop sizes are formed during dispensing. Prior to each fill or dispense operation, the microplate, reservoir, etc., to be used is positioned on a platform that raises it below the ends of the needles to the height needed for the vessel selected with a positional accuracy of $6.5 \mu \mathrm{m}$. The precision of the platform-positioning system is required for delicate operations including dispensing onto membranes and agar plates and for "touch off" of drops into dry plates.

To operate the system, a single "practice" run is performed to program the unit. The dispense volume, fill volume, wash volume and cycles and platform vertical motion are selected by push buttons and stored in one of 16 separate files in nonvolatile memory. An LED display on the front of the unit allows the operator to scroll through the files to confirm or

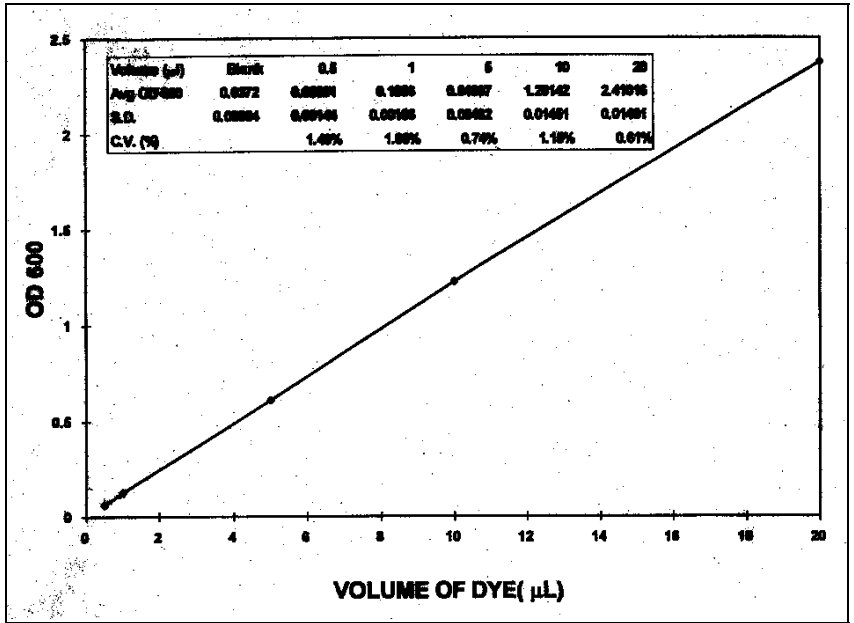

Figure 2. Dispensing uniformity of the Hydra-96. Using a concentrated dye solution as a detection reagent, the dispensing uniformity of the instrument was measured when delivering between 0.5 to $20 \mu \mathrm{L}$. 
edit parameters prior to operation. Dispense, fill, wash and empty functions are activated through push buttons on the front of the unit. An RS232 port on the side of the unit is used to control the instrument by a remote computer using a simple ASCII communication protocol.

\section{Measurement of Dispenser Uniformity}

Syringes were filled with a concentrated solution of naphthol blue black (Sigma Chemical, St. Louis, MO, USA) and volumes from 0.5 to $20.0 \mu \mathrm{L}$ dispensed into a series of 96well microplates containing $100 \mu \mathrm{L}$ of distilled water per well. After filling the syringes and prior to dispensing into the microplates, a few dispenses were normally performed back into the dye reservoir. The microplates containing the dye/water solution were mixed by hand using an 8-channel pipet and then read on a Molecular Devices THERMOmax ${ }^{\mathrm{TM}}$ plate reader (Sunnyvale, CA, USA). Data are presented after subtraction of values from a blank plate containing water only.

\section{Effectiveness of Bleach Treatment to Remove Residual DNA}

Preparation of DNA. Human genomic DNA was prepared from nuclei from $1 \mathrm{~mL}$ of whole blood from a DRB1-0301 homozygote using a modification of an ammonium acetate salting-out procedure (1). Final DNA preparation had an $\mathrm{OD}_{260 / 280}$ of $>1.8$. A segment of the DRB1 locus was then amplified by PCR for 30 cycles under standard conditions with DRB1-specific primers (2) using a Perkin-Elmer Model 9600 DNA Thermal Cycler (Norwalk, CT, USA) and thin-wall Strip-Ease ${ }^{\circledR}$ reaction tubes (Robbins Scientific). The amplified product was then denatured in $1 / 5$ vol $2 \mathrm{~N}$ $\mathrm{NaOH}$, then neutralized with 4/5 vol of $3 \mathrm{M}$ sodium acetate. The final solution of denatured DNA used for blotting was $0.22 \mu \mathrm{g} / \mu \mathrm{L}$.

Dot blot preparation. Dot blots were prepared with the Hydra-96 using the DNA preparation described above. Syringes were filled with $50 \mu \mathrm{L}$ of the DNA solution, except syringes $\mathrm{A} 1, \mathrm{~B} 1$ and $\mathrm{C} 1$, which contained a dilution series of the DNA, and syringe D7, which contained water only. A series

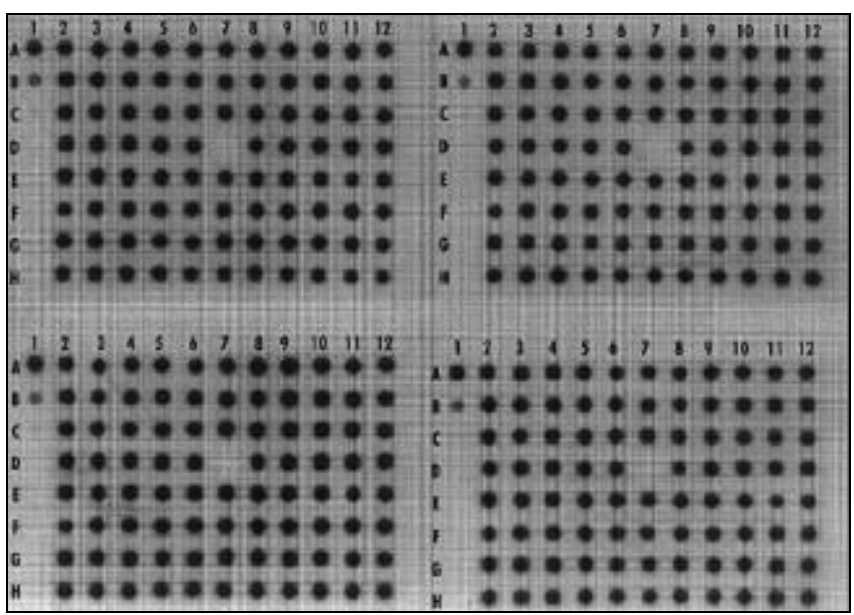

Figure 3. Uniformity of dot blots prepared with the Hydra. Four identical dot blots were prepared with the instrument, probed and autoradiographed overnight. Positions A1, B1 and C1 were serial dilutions of the DNA sample containing 220, 44 and 9 ng of DNA, respectively. Position D7 is a negative hybridization control. of four identical blots were prepared by dispensing $2 \mu \mathrm{L}$ of the solution per syringe onto Hybond ${ }^{\mathrm{TM}}-\mathrm{N}$ membranes (Amersham, Arlington Heights, IL, USA). The syringes were emptied and then rinsed with three up-and-down cycles with $2 \%$ bleach in water followed by three cycles with distilled water. The syringes were then refilled with distilled water and a dot blot prepared as described above, except positions A1, B1 and $\mathrm{C} 1$ contained the dilution series of DNA used in the first series of blots as hybridization controls. The syringes were then emptied again and rinsed only with distilled water for three cycles; then another dot blot was prepared. This process was repeated twice more, generating a total of four blots that would contain residual DNA left in the syringes.

Dot blot hybridization. Membranes were baked at $80^{\circ} \mathrm{C}$ for $2 \mathrm{~h}$, then prehybridized for $2 \mathrm{~h}$ at $57^{\circ} \mathrm{C}$ in a solution containing $6 \times$ sodium chloride sodium phosphate EDTA (SSPE), $5 \times$ Denhardt's, $0.5 \%$ sodium dodecyl sulfate (SDS) and 100

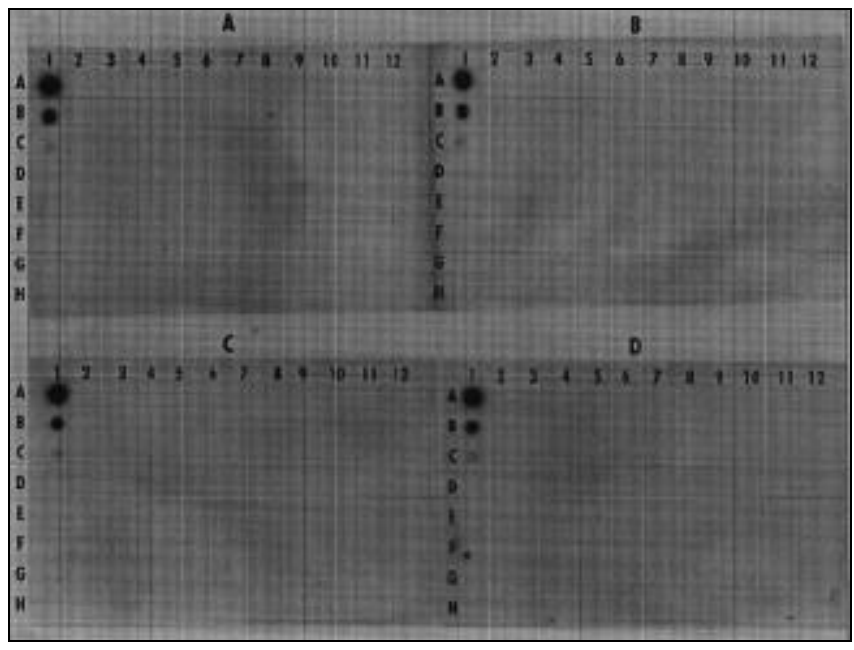

Figure 4. Effectiveness of bleach treatment to remove residual DNA. Dot blots were prepared after removal of the samples used in Figure 3 and rinsing with dilute bleach (A) and after each of three sequential rinses with water (B-D). Positive and negative hybridization controls are as described in Figure 3.

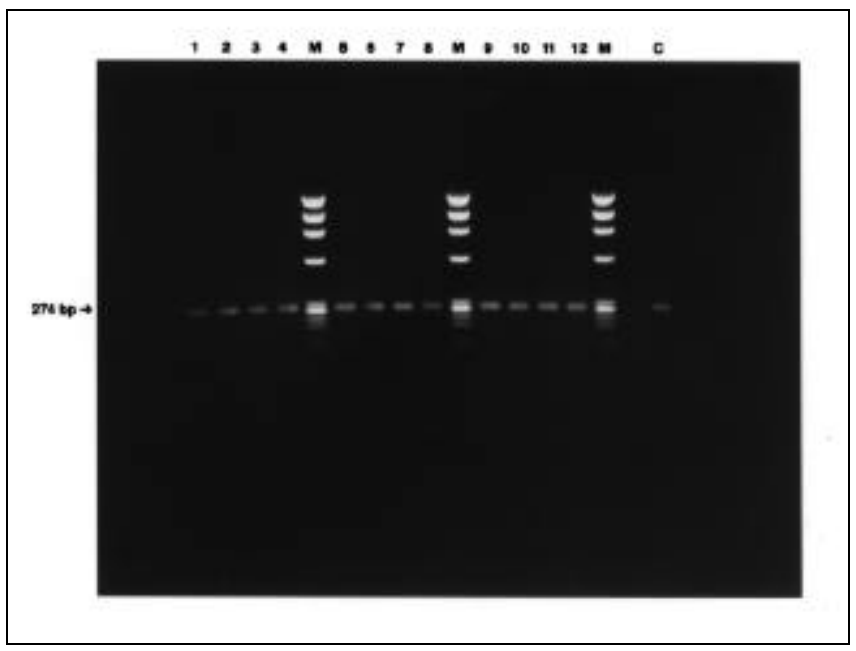

Figure 5. Effect of bleach treatment on subsequent PCRs. Reaction conditions are described in the text. Lanes 1-4 are identical reactions prepared from distilled water rinse \#1, lanes 5-8 from rinse \#2 and lanes 9-12 from rinse \#3. Positive control is shown in lane C. Molecular weight markers (lanes marked M) are an EcoR1 digest of $\phi X 174$. 
$\mu \mathrm{g} / \mu \mathrm{L}$ denatured herring sperm DNA (2). Blots were then hybridized in the same solution for an additional $4 \mathrm{~h}$ at $57^{\circ} \mathrm{C}$ using ${ }^{32} \mathrm{P}$-end-labeled DRB 7004 (2) as probe. After, the blots were washed twice in $2 \times \mathrm{SSPE} / 0.1 \%$ SDS and then $3-5$ times in $6 \times \mathrm{SSPE} / 1.0 \% \mathrm{SDS}$ at $60^{\circ} \mathrm{C}$. Following air drying, the blots were exposed to $\mathrm{X}$-ray film overnight at $-70^{\circ} \mathrm{C}$ with intensifying screens.

\section{Effect of Bleach Treatment on Subsequent PCRs}

The three distilled water solutions used to rinse the syringes described in the previous section were used in the preparation of a series of PCRs. Reactions conditions were as follows: $13 \mu \mathrm{L}$ of $\mathrm{H}_{2} \mathrm{O}$ containing $0.3 \mu \mathrm{g}$ human DNA, 15.2 $\mu \mathrm{L}$ of amplification mixture (containing $2 \mathrm{U}$ of Taq DNA Polymerase [Perkin-Elmer], nucleotides, $\mathrm{MgCl}_{2}$ and DRB1 primers) and $31.8 \mu \mathrm{L}$ of the distilled water rinse solutions. Positive control reactions contained pure distilled water in place of the rinse solutions. PCR was performed for 30 cycles, and the amplified products were separated on a $1.5 \%$ agarose gel and stained with ethidium bromide.

\section{RESULTS}

\section{Dispensing Uniformity}

Figure 2 shows the results of studies to measure the dispensing uniformity of the syringe array when delivering from 0.5 to $20.0 \mu \mathrm{L}$. Data presented are from analysis of datapoints from the individual wells across each microplate used for each volume evaluated. Results show that the volume delivered as measured by the dye concentration in the wells is a linear function of the volume selected on the instrument. Statistical analysis of the OD readings from the wells of each plate reveals a CV in the volume delivered of under $2 \%$ across the syringe array at all volumes tested. As another indication of the uniformity of dispensing, four identical dot blots were prepared using the instrument and then probed (Figure 3). The signal intensity is remarkably uniform from dot to dot within and between blots.

\section{Effectiveness of Bleach Treatment to Remove Residual DNA}

Figure 4 shows dot blots prepared after each of four sequential rinses of the syringes as a means of detecting residual DNA in the system. No DNA was found within the detection limits of the technique after a single rinse with $2 \%$ bleach and distilled water (Figure 4A) or in each of three successive rinses with distilled water only (Figure 4, B-D). The effectiveness of a similar rinsing protocol for removal of M13 template DNA from the syringes as measured by the absence of signal on an automated DNA sequencing instrument following cycle sequencing of syringe rinse solutions has also been reported (E. Mardis, Washington University Genome Sequencing Center, personal communication).

\section{Effect of Bleach Treatment on Subsequent PCR}

A series of PCRs were perfomed using the three distilled water rinses used after bleach treatment to determine if any residual bleach may be present in the syringes that would affect subsequent PCRs assembled using the Hydra-96. Gel 
electrophoresis of the PCR products (Figure 5) shows that when $30 \mu \mathrm{L}$ of the water rinses are used in place of distilled water in the PCRs, there is no effect on the quantity of PCR product produced relative to the controls. This study shows that simple rinsing of the syringe array with water effectively removes residual bleach. The bleach-rinsing protocol described can safely be used for cleaning the Hydra-96 when used for dispensing DNA solutions.

\section{DISCUSSION}

The Hydra-96 Microdispenser was developed to meet the specialized needs of laboratories that require high-speed, precise, repetitive dispensing of microliter volumes of reagents into microplates, PCR plates and filter membranes. Studies in this initial report have focused on examining the instrument's ability to uniformly deliver volumes down to $0.5 \mu \mathrm{L}$ and the effectiveness of rinsing the syringes to remove any residual sample. Results have shown that the instrument has less than $2 \%$ variance in dispensing volume, a performance that meets the requirements of the most demanding applications. Also shown is that simple rinsing of the syringes with dilute bleach effectively removed DNA that might have contaminated downstream reactions and that rinsing with distilled water removes residual bleach that might have interfered with subsequent PCRs prepared with the instrument. These results show that the instrument can be a useful tool in a variety of PCRbased applications. It further suggests that disposable tips are not necessary to prevent sample carryover, a feature that provides an important cost-saving advantage over all known multichannel, automated, liquid-handling devices.

Because of its unique characteristics, the Hydra-96 has found uses in a variety of applications in diverse laboratory environments. Drug-development laboratories involved in screening large combinatorial libraries use this instrument for dispensing small volumes of candidate compounds, detection reagents and other precious reagents. Human genome analysis laboratories use this instrument for preparing PCR and cycle sequencing reactions and for preparation of DNA sequencing templates. The Hydra-96 is also being used under sterile conditions for dispensing reagents into cell cultures used in various bioassays and for removing and adding media in micro-cell cultures used in single-cell-cloning techniques.

\section{REFERENCES}

1.Miller, S.A., D.D. Dykes and H.F. Polesky. 1988. A simple salting out procedure for extraction of DNA from nucleated cells. Nucleic Acids Res. 16:1215.

2.Tsuji, K., M. Aizawa and T. Sasazuki (Eds.). 1991. Proceedings of the Eleventh International Histocompatibility Workshop and Conference. Oxford University Press, Oxford, UK.

Address correspondence to James E. Stanchfield, Robbins Scientific Corporation, 814 San Aleso Avenue, Sunnyvale, CA 94086, USA. 